\title{
Probabilistic modeling for dynamic processes
}

\author{
Andrey Nesmiyan ${ }^{1}$, Lyudmila Kravchenko ${ }^{2, *}$, Vladimir. Khizhnyak ${ }^{1}$ and Elena Zubrilina ${ }^{2}$ \\ ${ }^{1}$ Azov-Black Sea Engineering Institute, branch of the Don State Agrarian University, 21, Lenina, \\ 347740, Zernograd, Russia \\ ${ }^{2}$ Don State Technical University, 1, Gagarin sq., 344003, Rostov-on-Don, Russia
}

\begin{abstract}
The probability of event occurrence can be determined by calculation, however for complex processes, accounting for multiple states is so complex that the probability of the final event cannot be determined differently than by collecting statistical data. This reduces the ability to predict outcomes in any process simulation, which affects the quality of advanced systems. The development of methods for predicting the probability of occurrence of an event when considering complex dynamic processes is a pressing task, the solution of which will help to improve the quality of modeling of technological processes and, as a result, to increase the efficiency of designing machines implementing them. The authors proposed a probability modeling method for dynamic processes, which is a special way of applying probability theory and is workable if the change in the parameters analyzed corresponds to the normal law of distribution. A study carried out on the analysis of the process of sowing corn seeds by vacuum sowing made it possible to predict with reliability more than $95 \%$ the probability of formation of "zero seed fodders" depending on their physical and mechanical properties, as well as parameters and adjustment modes of operation of the device.
\end{abstract}

\section{Introduction}

Almost all of the processes around us are probabilistic. The probabilistic occurrence of an event can be determined by calculation. However, for most complex processes, accounting for the total number of states is so complicated that the probabilistic occurrence of a final event cannot be determined otherwise than based on the collection of statistical data $[1,2,3]$. This narrows the possibility of predicting final results when modeling any processes, which ultimately affects the quality of functioning of developed systems [4].

The need for a practical solution of probabilistic problems led to the emergence and growth of a large number of «branches» of theory of probability and mathematical statistics: theory of reliability, game theory, concept of mass service, theory of optimal decision, theory of Markoff processes, etc. [4, 5, 6, 7]. It is especially difficult to analyze dynamic processes when the application of technical means proceeds under stochastically changing external conditions, while simultaneously varying the technological properties of the processed materials or the media used. This is clearly manifested during the operation of tunneling, digging and particularly agricultural machinery $[8,9,10,11$, etc.]. It is

\footnotetext{
*Corresponding author: lusya306@yandex.ru
} 
obvious that such technological indicators as the current value of the hourly fuel consumption when performing a particular operation; motor load level; traction resistance of a tool or machine; level of grain separation in a threshing drum of a combine harvester; level of grain loss behind the header, separator of small or rough grain ears of a combine harvester; applied dose of fertilizers in the field; depth of soil cultivation or sowing seed; presence and magnitude of damage to the grain; amount of wear of the working bodies; angular orientation of the cultivator shovels (and hence the gushing of the soil and traction resistance); presence or absence of an arch in the bunker of an agricultural machine and many others are probabilistic in nature and are determined by a combination of a large number of factors. As a rule, in determining the values of the indicators listed, a significant role is attributed to the dynamic characteristics of the analyzed processes.

In this regard, it can be argued that the development of techniques to predict the probability of occurrence of an event is an urgent task, when considering complex dynamic processes. The solution of the problem will help to improve the quality of modeling for technological processes and result in increasing the efficient designing of the machines that implement them.

\section{Materials and methods}

In most hypothetical tasks, creating conditions aimed at obtaining the desired result does not cause difficulties. However, in practice, developers have to deal with compromise tasks described by minimax models [12], in which, in addition to the main condition, one or more additional conditions apply. For example, to ensure maximum reliability of a machine with a given cost price, or to achieve its maximum performance with limited material and energy costs. In such conditions, developers often have to choose compromise values for individual parameters of a designed machine, for example, geometric and strength characteristics of working bodies and frame structures, assuming that external factors do not add up to a set of conditions that could damage the machine or disrupt the quality of its operation.

When implementing most dynamic processes, the probability of their violation or failure of a component is determined by the current values of the sum or ratio of forces acting on it. During the operation of the component, the value of these forces (force) usually varies significantly. There are three possible scenarios:

- the maximum possible value of the sum (or ratio) of the active forces is less than the permissible (boundary) value $\left(\sum F_{i \max }>\left[\sum F_{i}\right]\right)$. In this case, the performance of the component (event A) can be considered highly reliable ( $\mathrm{PA}=100 \%)$

- the minimum possible value of the sum (or ratio) of active forces is greater than the permissible (boundary) value $\left(\sum F_{i \min }>\left[\sum F_{i}\right]\right)$. In this case, the failure of the component (event $\mathrm{B})$ can be considered reliable $(\mathrm{PB}=100 \%)$;

- the permissible (boundary) value of the sum (or ratio) of active forces is greater than their minimum possible current value, but less than the maximum value $\left(\sum F_{i \min }<\right.$ $\left.\left[\sum F_{i}\right]<\sum F_{i \max }\right)$.

The third of the cases considered is as a rule the most common. The determination of the probability of the occurrence of one of the mutually exclusive events A or B (we mean the third case, but not events A or B) is most interesting from the point of view of practical use, and at the same time it is most technically difficult.

We have proposed a technique that allows at the known values $\sum F_{i \text { min }}, \sum F_{i \max }$ и $\left[\sum F_{i}\right]$ and with sufficiently high reliability to determine the probability of violation of the working process of a component. It should be noted that this technique is a fairly simple private way of applying theory of probability and is functional only if there is reason to believe that a change in the current value of the sum (ratio) of active forces submits to the 
normal distribution law. Moreover, the accuracy of the obtained result depends on how reliable the values $\sum F_{i \max }$ are received by an experimental or theoretical way.

The technique is as follows:

1. The boundary condition of a machine component is determined by calculation and experiment (for example, $\sum F_{i}<\left[\sum F_{i}\right]$ ).

2. Based on theoretical calculations or statistical data collection, the maximum and minimum values of the sum (or ratio) of active forces $\left(\sum F_{i \text { min }}, \sum F_{i \text { max }}\right)$ are determined. At the same time, the technique of using experimental data is more reliable, not only because it is closer to practice, but also because the presence of a set of data makes it possible to test the hypothesis about the normal law of distribution of the values of the sum of active forces.

3. The approximate average value of the sum (or ratio) of active forces is calculated.

$$
\sum F_{i c p} \approx 0,5\left(\sum F_{i \min }+\sum F_{i \max }\right)
$$

4. The approximate value of the root mean square deviation of the values of the sum (or ratio) of the active forces is calculated.

$$
\sigma_{F_{i}} \approx\left(\sum F_{i \max }-\sum F_{i \min }\right) / 6
$$

5. Using a well-known expression describing the law of normal distribution, the probability of maintaining the functionality of the machine component state is determined.

$$
p_{0}=\int_{\sum F_{i \min }}^{\left[\sum F_{i}\right]} \frac{1}{\sqrt{2 \pi} \cdot \sigma} e^{\frac{-\left(\sum F_{i}-\sum F_{i c p}\right)^{2}}{2 \sigma^{2}}} d \sum F_{i}
$$

We suggest we consider the example of using the proposed technique in the analysis of the working process of a vacuum seeding mechanism of a row crop drill [13].

The modern market is represented by agricultural seed drills of the following producers [14]: Hatzenbichler (Austria); Stanhay (England); Crucianelli (Argentina); Lidagroprommash (Belarus); Lidselmash (Belarus); Amazone (Germany); Fahse (Germany); Franz Kleine (Germany); Horsch (Germany); Kverneland-Accord (Germany); Rabe (Germany); Rau (Germany); Schmotzer (Germany); Kongskilde (Danmark); Moldagrotechnics (Moldova); Agricola Italiana (Italy); Mascar S.p.A (Italy); Mashio Gaspardo (Italy); Mater Macc (Italy); Sfoggia (Italy); BDM-agro (Russia); Belgorod factory «Rhythm» (Russia); Millerovoselmash (Russia); Technique-service (Russia); White (USA); Amity Technology (USA); Case IH (USA); Great Plains (USA); John Deere (USA); Kinze (USA); Massey Ferguson (USA); Achtyrselmash (Ukraine); Todak (Ukraine); Chervona Zirka (Ukraine); Kuhn (France); Monosem (France); Quivogne (France) and the others. Seed drills of various producers significantly differ from each other in terms of design, while one of their most important classification features is the principle of seed dosing, which is carried out by seeding mechanisms. Modern seed drills carry out seed dosing with vacuum seeding mechanisms, sowing device of overpressure or mechanical mechanisms. In the Russian Federation and European countries, seed drills with a vacuum metering device (seeding mechanism) are most popular [13].

The technological function of seeding mechanisms lies in one-seeded feeding from total seed mass and dumping seeds at approximately equal intervals into the furrow prepared by the drill coulter. In the vast majority of vacuum seeding mechanisms, this process proceeds as follows: the seeds (Figure 1) from the hopper 1 enter seed the chamber 2, where under the action of the agitator 3 and the rarefaction created in the vacuum chamber 4, they are captured by the suction holes of the sowing disk 5 mounted on the drive shaft 6 . When the shaft rotates, the seized seeds are transferred to the area of the 
reflector of «extra» seeds 7 , which shifts them from the center of the suction holes to the radial direction, due to which the extra seeds are discharged into the seed chamber 2, while the main seeds, most centrally located at the suction holes, are delivered by the sowing disk 5 to the lower part of the seeding mechanism, where because of rarefaction cut-off they result in separating from the suction holes by the release guiding mechanism 8 and then, being under gravity, they fall into the furrow formed by the drill coulter (not shown in Figure 1) [14].

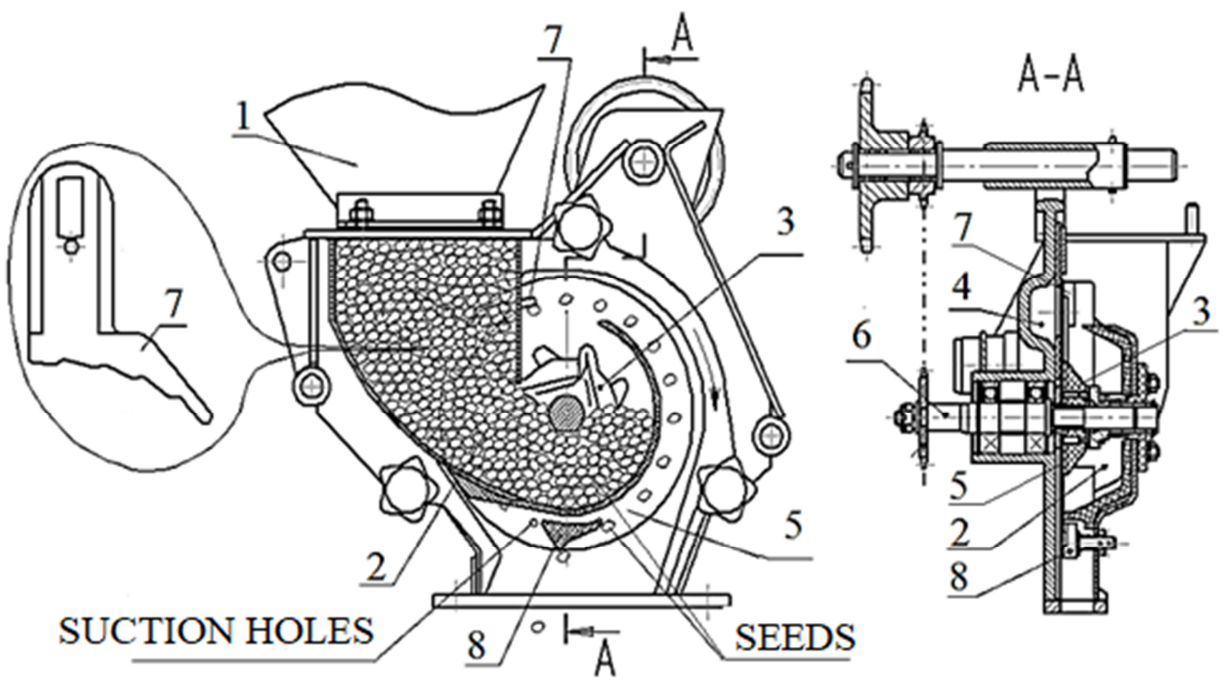

Fig. 1. Diagram of a vacuum seeding mechanism of a row-crop drill (on the example of a seeding mechanism of the row-crop drill MS-8. Manufacturer: JSC «Millerovoselmash», Russia, Millerovo, Rostov Region).

The formation of a one-seeded feed is a complex dynamic process in which the final result is affected by a significant number of such factors as: geometric parameters of seeds, their mass, frictional and aerodynamic properties; diameter of suction holes; depth of the vacuum in the vacuum chamber; linear speed of the centers of the suction holes, determined by planned speed of the seeding mechanism and seeding amount; position of the reflector of «extra» seeds relative to the centers of the suction holes; thickness of the seed layer in the seed chamber; seed disc thickness; number of suction holes on the sowing disk, as well as the radius of their centers on it, etc. [11, 14].

Most experts agree that the basic stage in the operation of a vacuum seeding mechanism, which largely determines the course of all subsequent ones, is the seizure of seeds by suction holes from their total mass located in the seed chamber [14]. Moreover, we can assume that the i-th seed was fixed at the suction hole if the current value of the resultant resistance forces Ri(t) (Figure 2), oriented in a plane parallel to the plane of the sowing disk (harmful force), does not exceed the peak value [Fi](t) of the friction force Fi (t) of the surface of the sowing disk on the seed (useful force) $[11,14]$.

When calculating the current value of the resultant resistance forces, the following components are traditionally taken into account (Figure 2): friction force $\bar{F}_{\mathrm{pss} i}$ of the adjacent layer of seeds on the seized seed; vertical pressure force of the overlying seed mass $\bar{F}_{\mathrm{vd} i}$; resistance force $\bar{F}_{\mathrm{c} i}$ of the seed mass lying on the trajectory of the seized seed; force of seed weight $\bar{F}_{\text {Ti }}$; force of its inertia $\bar{F}_{\text {ini }}$; centrifugal force $\bar{F}_{c i}$. Trying to simplify calculations, most authors conditionally refer force to support the seized seed by the agitator blades $\bar{F}_{\mathrm{pvi}}$ to the resistance forces $[11,14]$.

When a seed drill operates under production conditions, a seeding mechanism and seeds 
located in it are additionally affected by force of inertia due to the micro- and mesorelief of the field surface. However, in this study an experimental performance evaluation of the seeding mechanism was carried out in laboratory conditions, where these forces are absent. Therefore, when calculating, the «field relief» forces of inertia were also neglected.

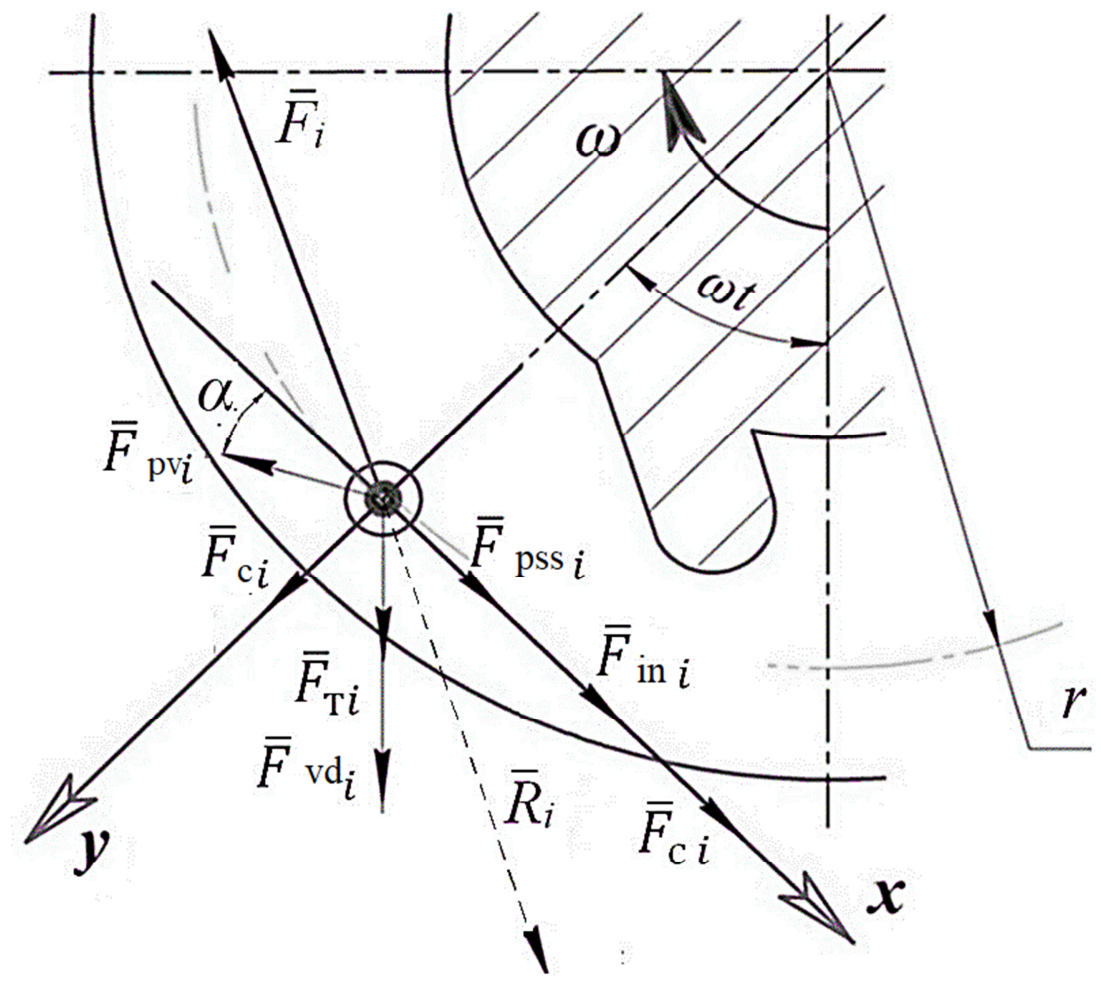

Fig. 2. Diagram of forces acting on a seized seed in a seed mass.

The values of the components of the resultant resistance force are determined by known techniques.

Peak value [Fi] of the friction force of the surface of the seeding disc on the seed [11, $13,14]$.

$$
\left[F_{i}\right](t)=\mu(t)\left(F_{\mathrm{vd} i}(t) \cdot \operatorname{tg} \beta(t)+F_{\mathrm{pp} i}(t)\right)
$$

where $\mu(t)$ is the current value of the friction coefficient of the surface of the seeding disk on the seed;

$\beta(t)$ is the current value of the angle of inclination of the force lines (direction of the flow of force in bulk material), deg.;

$F_{\mathrm{pp} i}(t)$ is the current value of the suction force (oriented normally to the plane of the sowing disk, therefore, it is not displayed in Figure 2), N.

The force of vertical pressure of the overlying seed mass can be determined by calculation, taking into account changes in the seed length $[11,13,14]$

$$
F_{\mathrm{vd} i}(t)=\gamma \cdot\left(r \cos \omega t-h_{1}\right) S_{i}(t) \cdot g
$$

where $\gamma$ is volumetric mass of seed layer in a seed chamber, $\mathrm{kg} / \mathrm{m} 3$;

$r$ is radius of the centers of suction holes on the seeding disk, m;

$\omega t$ is angular movement of suction hole from a zero (lower) position, deg. 
$h_{1}$ is vertical distance between the axis of rotation of the drive shaft and the edge of the feed neck feed hopper, $\mathrm{m}$;

$S_{i}(t)$ is current value of the mid-section area of the seized seed, $\mathrm{m} 2$.

Using simplified calculations, it is possible to calculate with sufficiently high accuracy the suction force as a contact one, with its value in the classical theory of vacuum seeding mechanisms being assumed to be proportional to the area of the suction hole. However, such a situation can be observed only with constant complete overlapping of the suction hole with a seed. More recent studies have shown that as a result of passing the removed seed through the mass of the remaining seeds, there form voids behind them on the trajectory of their moving $[11,13,14]$. After closing, the seeds do not completely overlap it on the trajectory of the metering device in a projection and the formed overlap area $S_{\mathrm{p}}$ most often does not equal (less) to the area of the round metering device $S_{0}$.

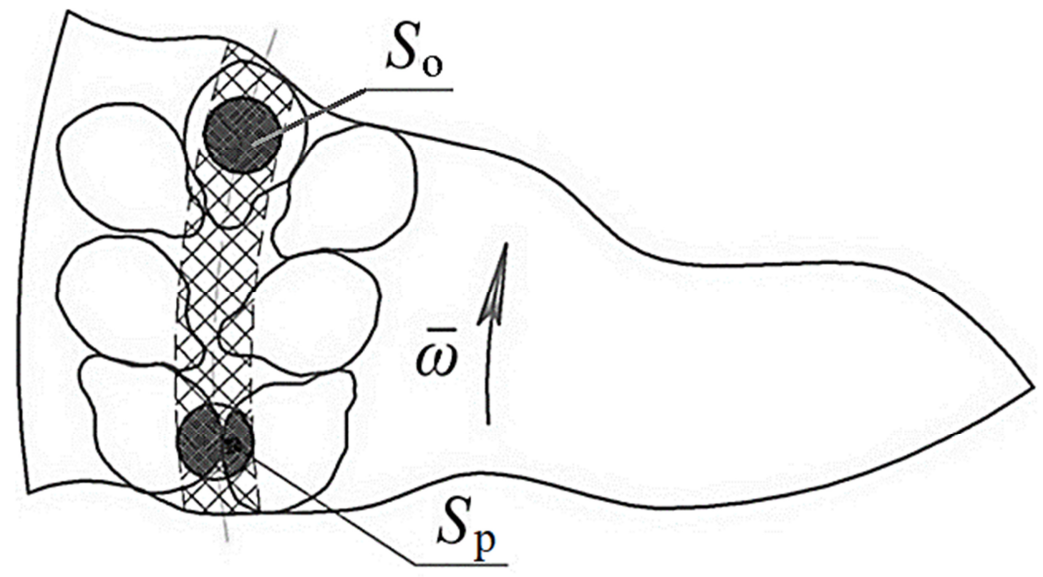

Fig. 3. Scheme of possible seed arrangement against suction holes.

In view of the above, the current value of the contact suction force is more logical to calculate using the expression $[11,13,14]$.

$$
F_{\mathrm{pp} i}(t)=k_{\mathrm{pp}} \cdot S_{\mathrm{p}}(t) \cdot H
$$

where $k n p$ is a coefficient taking into account air suction, $\mathrm{k} \Pi=0,7 \ldots 0,9[12,13]$;

$S_{\mathrm{p}}(t)$ is the current value of the area of overlapping of the metering device by a seed, $\mathrm{m} 2$;

$N$ is the amount of vacuum in the plane of the suction hole, $\mathrm{Pa}$.

The value of the overlap area $S_{p}(t)$ of the suction hole with a seized seed depends on the degree of mutual overlap.

$$
S_{\mathrm{p}}(t)=\int_{-0,5 d_{o} \sin \left(\arccos \left(0,5-C_{\text {по }}\right)\right)}^{0,5 d_{o} \sin \left(\arccos \left(0,5-C_{\text {по }}\right)\right)}\left(\sqrt{0,25 d_{o}^{2}-x^{2}}+\left(C_{\mathrm{po}}-0,5\right) d_{o}\right) d x,
$$

where $d_{0}$ is the diameter of the suction hole, $\mathrm{m}$;

$C_{\text {po }}$ is the degree of ovelap of the diameter of the suction hole with a seed.

The probability of seizing a seed by a suction hole depends on the ratio of the friction force of the surface of the seeding disk on the seed and the resultant resistance forces.

$$
\gamma_{i}(t)=\frac{\left[F_{i}\right](t)}{R_{i}(t)} .
$$

If $\gamma_{i}(t) \geq 1$, then the conditions necessary for seizing a seed can be considered as 
formed, i.e. the probability of zero feed will be zero $p_{0 i}=0=0$. This statement is based on the results of studies that proved that the seizure of a seed is the least likely in the first phase of this process, while the achievement of an instantaneous value $\gamma_{i}(t) \geq 1$ automatically contributes to the further stabilization of the seed at the suction hole [14]. $p_{0 i}=1$.

If at $\gamma_{i}(t)<1$ the seed is not seized, therefore the probability of «skipping» is equal to

We accept the assumption that the distribution of the values of the relation submits to the normal law. Such an assumption is valid, because in this case the desired value is formed under the influence of a large number of factors, among which none prevail [8]. We suggest we define the calculated minimum and maximum ratio $\frac{\left[F_{i}\right](t)}{R_{i}(t)}$.

$$
\begin{aligned}
& \gamma_{i}(t)_{\text {min }}=\frac{\left[F_{i}\right](t)_{\min }}{R_{i}(t)_{\max }} \\
& \gamma_{i}(t)_{\max }=\frac{\left[F_{i}\right](t)_{\max }}{R_{i}(t)_{\min }}
\end{aligned}
$$

Respectively:

$$
\gamma_{i}(t)_{\mathrm{sr}}=0,5\left(\frac{\left[F_{i}\right](t)_{\min }}{R_{i}(t)_{\max }}+\frac{\left[F_{i}\right](t)_{\max }}{R_{i}(t)_{\min }}\right) .
$$

The approximate value of the standard deviation of the values of the sum (or ratio) of the active forces is calculated.

$$
\sigma_{\gamma_{i}(t)}=\left(\frac{\left[F_{i}\right](t)_{\max }}{R_{i}(t)_{\min }}-\frac{\left[F_{i}\right](t)_{\min }}{R_{i}(t)_{\max }}\right) / 6 .
$$

In accordance with an expression (3), the probability of the zero feed formation.

$$
p_{0}=\int_{\gamma_{i}(t)_{\min }}^{1} \frac{1}{\sqrt{2 \pi} \cdot \sigma_{\gamma_{i}(t)}} e^{-\frac{\left(\gamma-\gamma_{i}(t) s \mathrm{sr}\right)^{2}}{2 \sigma_{\gamma_{i}(t)}{ }^{2}}} d \gamma,
$$

Taking into account expressions (1 ... 13), as well as well-known dependences, as an example, we calculated the probability of the formation of zero feed of corn seeds depending on the amount of rarefaction in the plane of the suction holes. The following values of the initial data for the calculation were taken: average seed length $a=10,61 \mathrm{~mm}$; average seed width $b=8,02 \mathrm{~mm}$; average seed thickness $c=4,79 \mathrm{~mm}$; root mean square deviations of seed sizes $\sigma a=0,70 \mathrm{~mm}, \sigma b=0,65 \mathrm{~mm}$ and $\sigma \mathrm{c}=0,71 \mathrm{~mm}$; calculated value of the angular velocity of the sowing disk $\omega=5.2 \mathrm{rad} / \mathrm{s}$; radius of the suction holes on the sowing disk $\mathrm{r}=75 \mathrm{~mm}$; outer radius of the seed agitator $\mathrm{rB}=60 \mathrm{~mm}$; sliding coefficient of seeds relative to the agitator blades $\tau=0,57$; diameter of suction holes $\mathrm{d} 0=5 \mathrm{~mm}$; maximum and minimum values of the angle of seed placement $\beta \max =50$ and $\beta \mathrm{min}=0$ deg.; friction coefficient of the surface of the seeding disk on the seed $\mu=0,73$; minimum seed weight $\operatorname{mmin}=0,06$; maximum seed weight $\operatorname{mmax}=0,45 \mathrm{~g}$; degree of overlap of the diameter of the suction hole with a seed $\mathrm{Cmin}=0,5, \mathrm{Cmax}=1$; average density of seed mass in the seed chamber $\gamma=740 \mathrm{~kg} / \mathrm{m} 3$.

Physical and mechanical properties of seeds were taken on the basis of generalized characteristics obtained be experiment $[11,14]$.

The results of the calculations are presented in graphical form in Figure 4. 


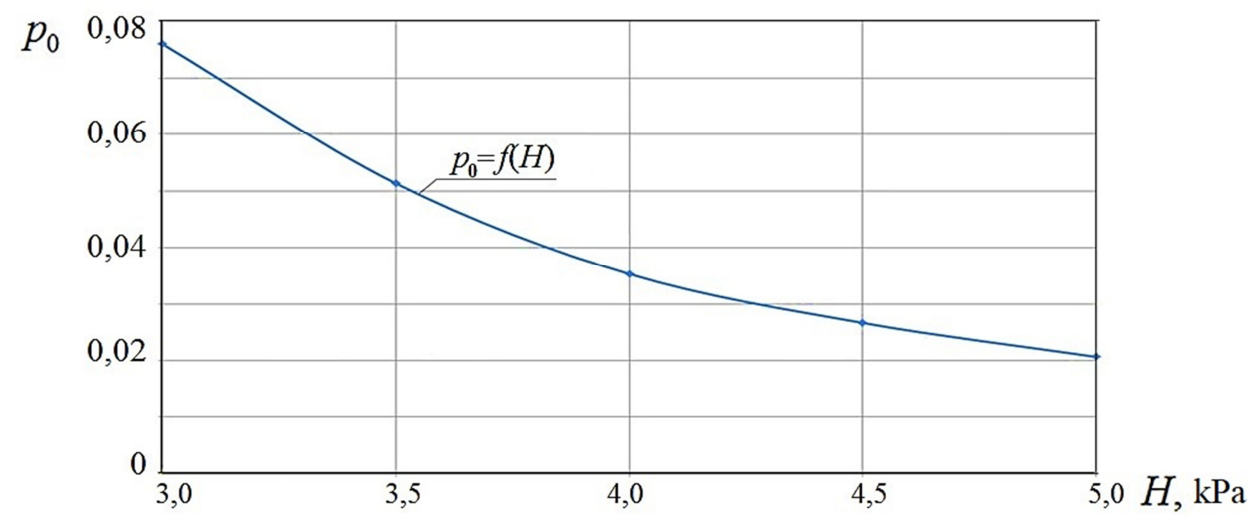

Fig. 4. Calculated dependence of the probability of zero feed formation of corn seeds with suction holes dependent on the rarefaction in a vacuum chamber.

The obtained results allow us to conclude that the proposed technique allows predicting the probability of the occurrence of an event when considering complex dynamic processes. In this case, this is the probability of holding corn seeds at the suction holes of the seeing disk. In addition, its application allows us to simulate the results of processes when changing a set of input data. In the case under consideration, there takes place change in the value of rarefaction, the rotation speed of the seeding disk, dimensional or mass characteristics of seeds, etc.

The dependence shown in Figure 4 corresponds to the accumulated experimental data. However, it is necessary to quantify a convergence of the results obtained using the proposed technique and experiments.

The verification of the convergence of the results obtained in various ways was carried out in three stages.

At the first stage, using the proposed dynamic modeling technique, the dependence of the probability of zero feed formation during sowing of corn seeds was obtained depending on the angular velocity of the seeding disk. When calculating the value of rarefaction in the vacuum chamber was assumed to be $4.8 \mathrm{kPa}$ (the recommended value is $4.5 \ldots 5.0 \mathrm{kPa}$ ). The values of the remaining factors were taken at the same level as before.

At the second stage, an experimental study of performance indicators (namely, the frequency of zero feed formation) was carried out with a vacuum seeding mechanism of a row-crop drill. The experiments were carried out on a computerized complex [14] (Figure 5 ) at the department «Technologies and Means of Mechanization of the Agro-Industrial Complex», the Azov-Black Sea Engineering Institute, branch of the Don State Agrarian University, Zernograd, Rostov Region). 


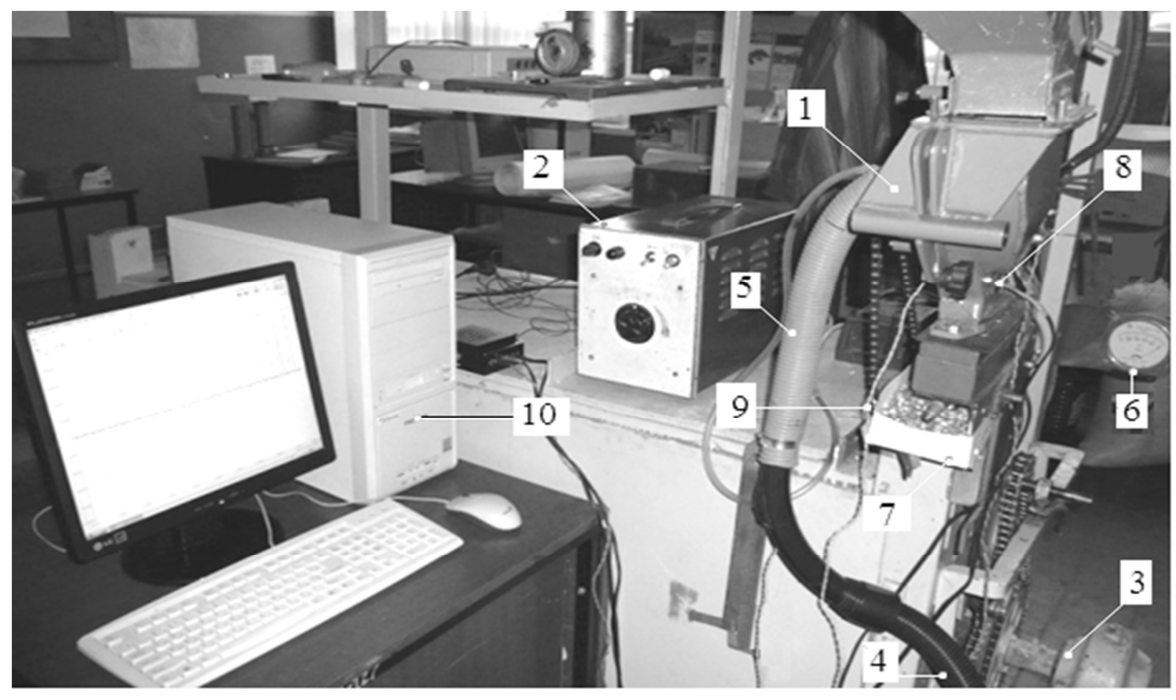

Fig. 5. Diagram of a computerized complex for investigating vacuum seeding mechanism. Where: 1 - investigated vacuum seeding mechanism; 2 - electric motor remote control (laboratory transformer); 3 - direct current electric motor; 4 - source of rarefaction (vacuum station); 5 pneumatic drive; 6 - vacuum gauge; 7 - seed collector; 8 - a set of sensors; 9 - screened cable; 10 computer with application program package.

In addition to the mentioned equipment, the digital metering equipment included a universal board for collecting analogue and input / output digital information LA-1,5PCI (hereinafter - analog-to-digital converter (ADC)); a set of sensors that monitor the performance of the studied seeding mechanism (for determining the total number of feeds, as well as the number of «skips»); interface card, providing the transmission of an analog signal from sensors to the ADC [14].

During the experiment, the angular velocity of the seeding disk varied in the range from 1.0 to $6.6 \mathrm{rad} / \mathrm{s}$ in increments of $1.4 \mathrm{rad} / \mathrm{s}$. The experiment was carried out in triplicate, in each of which three hundred seed feeds were recorded.

At the third stage, the theoretical (dependence of a in Figure 6) and experimental data (dependence of $\mathrm{b}$ in Figure 6) were compared using Pearson's criterion.

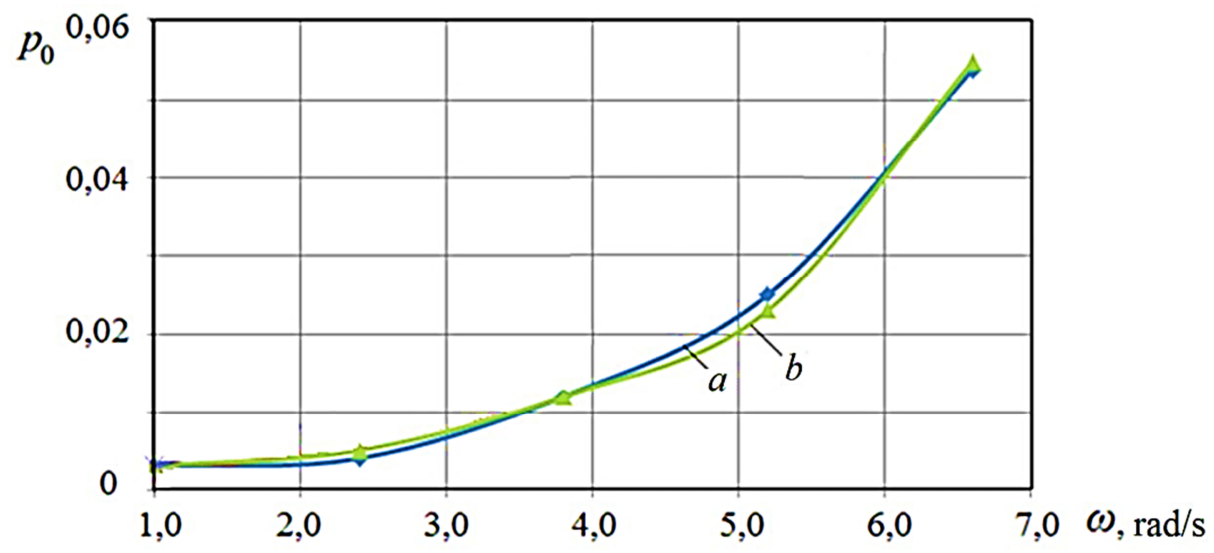

Fig. 6. Probability of zero feed formation of corn seeds with a mechanism depending on the angular velocity of the seeding disk. Where: $a$ - dependence obtained by calculation (probability of zero feed formation), $b$-dependence obtained by experiment (frequency of zero feeds). 
From the data in Figure 6 one can see that the proposed model of the seed feed process by the metering elements of the vacuum seeding mechanism allows predicting the probability of the formation of «skips» with sufficiently high accuracy. In the case under consideration, the calculated value of Pearson's criterion [1] when considering the process of removal of seeds from the seed chamber was $\chi 20 \mathrm{cc}=4 \cdot 10-4$. This is significantly lower than the table value $\left[\chi_{2}\right]=0,711$ with an error probability of 0.05 , which confirms the correspondence of the obtained model to the real process.

\section{Conclusion}

The development of a technique to predict the occurrence of an event when considering complex dynamic processes is an urgent task, the solution of which will help to improve the quality of modeling for technological processes and, as a consequence, increase the efficiency of designing machines that implement them. In this regard, the authors proposed a probabilistic modeling technique for dynamic processes, which is a particular way of applying theory of probability and is workable if a change in the analyzed parameters submits to the normal distribution law. Using the proposed technique, calculations are implemented in several stages: determining a boundary condition for the performance of the component; determination of the maximum and minimum values of the analyzed indicator; determination of its approximate mean value and standard deviation; calculation of the probability of the occurrence of a predicted event using the law of normal distribution. An analysis of the process of sowing corn seeds with a vacuum seeding mechanism revealed that the proposed technique of probabilistic modeling for dynamic processes made it possible to predict the probability of zero seed feeds formation depending on their physical and mechanical properties with a reliability of more than $95 \%$, as well as parameters and tuning modes of the mechanism.

\section{References}

1. J. Richard, L. Morris, An introduction to mathematical statistics and its applications (Pearson Education Inc., 2014)

2. L. Jay, Devore, N. Kenneth, Modern Mathematical Statistics with Applications (Springer New York Dordrecht Heidelberg, London, 2012)

3. V.A. Gushchin, A.I. Sukhinov, A.V. Nikitina, A.E. Chistyakov, A.A. Semenyakina, Computational Mathematics and Mathematical Physics 58(8), 1316-1333 (2018) DOI: 10.1134/S0965542518080092

4. L. Guzzella, Modeling and Analysis of Dynamic Systems, Course Notes (ETH, Zurich, 2011)

5. J.W. Simpson-Porco, F. Dorfler, F. Bullo, Nature Communications 7, 1079 (2016)

6. R. Bardenet, A. Doucet, C. Holmes, arXiv 1505, 02827 (2015)

7. M.S. Ross, Introduction to probability 784 (2010)

8. V.A. Chernovolov, V.A. Kravchenko, L.V. Kravchenko, A.Yu. Nesmiyan, V.I. Khizhnyak, S.A. Sherstov, Amazonia Investiga 17, 670-675 (2018)

9. A.I. Sukhinov, A.V. Nikitina, A.A. Semenyakina, A.E. Chistyakov, CEUR Workshop Proceedings 1576, 308-319 (2016)

10. I. Markvo, E. Zubrilina, V. Novikov, E3S Web of Conferences 126, 00054 (2019) 
11. A.I. Sukhinov, D.S. Khachunts, A.E. Chistyakov, Computational Mathematics and Mathematical Physics 55(7), 1216-1231 (2015) DOI: 10.1134/S096554251507012X

12. G.A. Wilson, Li Lin, Gao Zhen, Moan Torgeir, Ocean Engineering 1251, 308-327 (2016)

13. L. Jieun, A. Shigrekar, Nuclear Engineering and Design 348, 131-145 (2019)

14. T. Perez, Perspective IFAC 48, 217-222 (2019)

15. W. Cai, B. Wang, L. Longxiang, X. Zhou, An operable platform towards functionalization of chemically inert boron nitride nanosheets for flame retardancy and toxic gas suppression of thermoplastic polyurethane 1781, 10746 (2019) 